\title{
Der Mensch als natürliches und künstliches Wesen
}

\author{
AlEš HAVLÍčEK $\dagger$ \\ Jan Evangelista Purkyně University in Ústí nad Labem (Czech Republic)
}

Title

The Human Being as Natural and Artificial

\begin{abstract}
This article traces the heritage of the Aristotelian political investigations in Thomas Hobbes's Leviathan. It can be very important to note that the definition of man as a "social" animal will develop in Hobbes's characterization by the opposite terms, the "natural" man and man "artifact"; two adjectives that Hobbes uses instead of "political", the very Aristotelian adjective.
\end{abstract}

Key Words

Aristotels's Politics, Thomas Hobbes's Leviathan, "Social/Political" Animal

Recibido: 17/06/2015 - Aceptado: 11/12/2015

Aristoteles schreibt als einer der ersten Denker über die natürliche Bestimmung des Menschen, in einer Gemeinde zu leben, also über $\zeta \hat{\omega} o \nu$

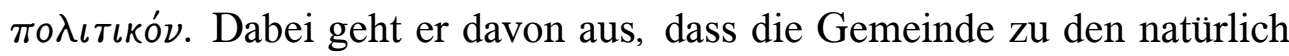
gegebenen Seienden gehört (tôn physei hê polis, 1253a 2). An diesen Gedanken knüpft in der Zeit der Aufklärung auch Thomas Hobbes an, wenn er vom Staat als einem „künstlichen Menschen“ („artificiall man“, Einleitung) spricht. Beide Denker setzen dabei einen natürlichen Zustand voraus, der für Aristoteles der Zustand des Vermögens oder der wesentlichen Möglichkeit (dynamis) ist, für Hobbes hingegen der Kriegszustand, dem man sich durch Vertragsabschluss entziehen muss. Für Aristoteles wird diese Natürlichkeit als Vermögen mittels der Kunst ( technê) erfüllt und verwirklicht, mit der jedes Individuum je nach seinem 
Geschick und seiner Befähigung unterschiedliche Stufen der Erfüllung erreicht. Dem gegenüber bewertet Hobbes diesen natürlichen Zustand als andauernden Krieg von Allen gegen Alle negativ und ersetzt ihn durch einen neuen, künstlichen Vertragszustand. Diese unterschiedlichen Auffassungen von Natürlichkeit, die nicht nur die Natürlichkeit des Menschen betreffen, sind der Ausgangspunkt unserer Überlegungen über den Menschen als „politisches Wesen“, wobei wir uns vor allem auf den oft betonten Unterschied zwischen den Staatsauffassungen von Aristoteles und Hobbes konzentrieren, das heisst auf die verbindenden und trennenden Wesenszüge der Entstehung und Konzeption des Staates.

Aristoteles schrieb über die ursprüngliche Philosophie, über Politik, Ethik, Logik, Natur, Meterologie, Rhetorik oder Poetik, aber in keinem seiner Texte befasst er sich ausführlich mit dem Menschen. In seiner Nikomachischen Ethik erscheint zwar einmal das Wort anthrôpologos, aber nur in der Bedeutung eines Menschen, der andere Menschen verleumdet ${ }^{1}$. Andererseits, finden wir aber an vielen Stellen seiner Texte unterschiedliche verstreute Äußerungen über den Menschen, wie zum Beispiel in der oben erwähnten Auslegung aus der Politik, dass er physei politikon zôon, d.h. durch seine Natürlichkeit zum Leben in der Gemeinde bestimmt sei ${ }^{2}$, oder auch die Behauptung, dass der Mensch logon echei, dass er also Sprache besitzt, die ihm wesentlich zugehört ${ }^{3}$.

Im Fall der ersten Äußerung sind die Interpreten über die Frage uneins, ob wir den Ausdruck politikon zôon in einem weiteren oder engeren Sinn $\mathrm{zu}$ verstehen haben. Die enger gefasste Variante besagt, dass nur der Mensch die Fähigkeit besitzt, in der Gemeinde zu leben, so dass nur er ein politisches Lebewesen ist und ein Gemeindebewohner werden kann.

1 Vgl. Aristoteles, Ethica Nicomachea (Eth. Nic.), IV 3,1125a 5. Vgl. O. HöFFE, Grundaussagen über den Menschen bei Aristoteles, «Zeitschrift für philosophische Forschung» 30 (1976), S. $227 \mathrm{nn}$.

Aristoteles, Politica(Pol.), I 1253a 3, III1278b 20, Eth. Nic. I 7, 1097b 12.

Aristoteles, Pol. I 1253a 9-10; VII1332b 5-6.

П Н Г Н / F O N S I (2016), 145-159 
Die weiter gefasste Interpretation begreift hingegen den Ausdruck politikon zôon nicht nur als Charakterisierung des Menschen, sondern auch einiger Herdentiere, wie zum Beispiel der Bienen, Wespen, Ameisen oder Kraniche ${ }^{4}$. Die Verfechter der erweiterten Variante, Autoren wie Wolfgang Kullmann oder John M. Cooper ${ }^{5}$, berufen sich nicht nur auf die Passage 1253a 8-9 in der Politik, wo es heißt, der Mensch sei politikon zôon in einem größeren Maß oder auf einer höheren Stufe $^{6}$ als Herdentiere oder Bienen, sondern auch auf eine Stelle in der Historia animalium ${ }^{7}$, aufgrund derer sie zôn politikon als biologische, und nicht als politische Beschreibung verstehen.

Auf die Passage 1253a 6 in Aristoteles' Politik beruft sich auch Hobbes in seinem Werk Leviathan, wenn er sich im XVII. Kapitel die Frage stellt, warum bestimmte, „sociably“ lebende Lebewesen wie zum Beispiel Bienen und Ameisen, die weder über Verstand noch Sprache verfügen, ohne Nötigung in Gesellschaft leben. Bevor er auf diese Frage

4 Aristoteles, Pol. I 1253a 7-9; Metaphysica(Met.), VII 1029a 29.

5 Vgl. W. Kullmann, Der Mensch als politisches Lebewesen, «Hermes» 108 (1980), S. 419-443; J. M. Copper, Political Animals and Civic Friendship, in: G. Patzig (ed.), Aristoteles' "Politik“, Göttingen 1990, S. 221-242. J. M. COOPER, Political Community and the Highest Good, 2009 (Sonderdruck).

6 Aristoteles, Pol. I 1253a 7-8: politikon ho anthrôpos zôon pasês ... zô̂u mallon (engl.: „a greater measure“, dtsch. : „in weit höherem Masse“.

7 Aristoteles, Historia animalium (Hist. animal.) I 487b 32-488a 12: „Ein Teil der Tiere

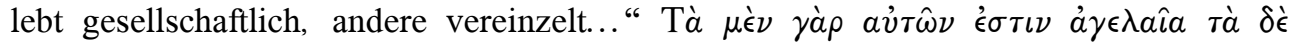

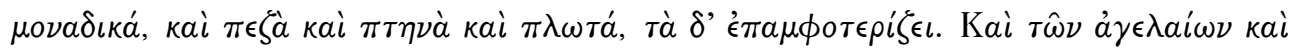

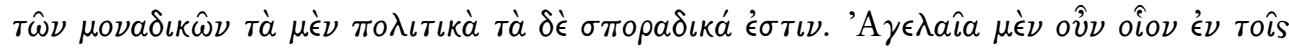

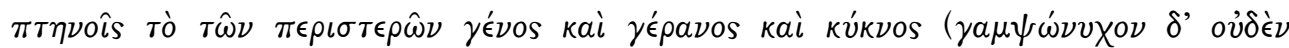

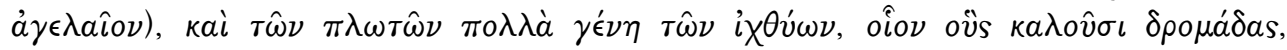

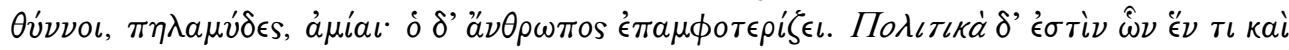

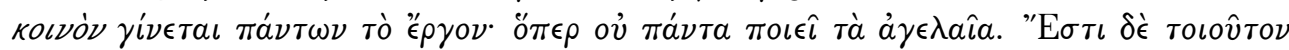

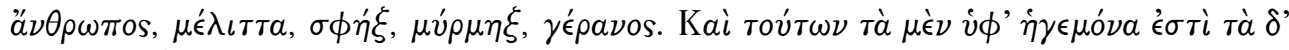

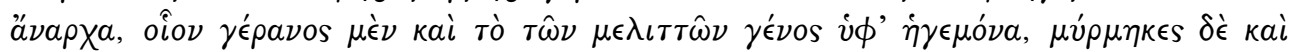

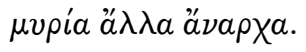


in der Form von sechs Vergleichen zwischen Menschen im Besonderen und Lebewesen im Allgemeinen Antwort gibt, bezeichnet er diese Lebewesen als „political creatures“, die im Unterschied zu den Menschen nicht in der Lage seien zu sagen, was sie als „common benefit“ erachten.

Wenn wir nun auf die oben angeführten Interpreten Kullmann und Cooper zurückkommen, so vermuten wir im Unterschied zu ihnen, dass es sich in der Politik oder in der Historia animalium nicht um eine biologische, sondern um eine dezidiert politische Beschreibung handelt. Denn unter der Voraussetzung, dass der Mensch physei politikon zôon also ein Lebewesen ist, dass durch seine Natürlichkeit dazu bestimmt ist, in der Polis zu leben, bezieht sich Aristoteles sowohl auf dessen Existenz als auch auf sein Verhalten (politikon ist ein Adjektiv, das den Bezug zur polis, zur Gemeinde ausdrückt). In seinem Sinne können wir sagen, dass die Gemeinde zwar wegen des Lebens und zu seiner Erhaltung entsteht, dass sie aber für ein gutes Leben weiter besteht ${ }^{8}$. Auf der Grundlage der Analogie zwischen der Eudaimonie der Polis und des Individuums ${ }^{9}$ kann auch über den Menschen ausgesagt werden, dass er zunächst bestrebt ist, sein Leben $\mathrm{zu}$ erhalten, um dann aber ein gutes Leben zu führen. Und gerade ein gutes Leben ist das Ziel und der Sinn jedes einzelnen menschlichen Lebens und auch der Gemeinde. Einen ähnlichen Gedanken finden wir auch in der Historia animalium, wo zôn politikon als ein Lebewesen charakterisiert wird, dem es um eine „gemeinsame Aufgabe“ (koinon ergon) ${ }^{10}$ geht, was bedeutet, dass ihm das Allgemeinwohl am Herzen liegt. Diese Schlussfolgerung bezüglich des Allgemeinwohls stützen wir auf eine die Klassifizierung der einzelnen Verfassungen betreffende Passage im Buch III. der Politik, in dem vom Königtum, der Aristokratie und der politeia die Rede ist. Aristoteles führt

8 Aristoteles, Pol. I $1252 \mathrm{~b} 30$.

9 Vgl. P. WEBER-SCHÄFER, Einfuihrung in die antike politische Theorie, II, Darmstadt 1967, S. $37 \mathrm{nn}$.

10 Aristoteles, Hist. animal. I 488a 7. 
hier aus, dass der Unterschied zwischen der Tyrannis, der Oligarchie und der Demokratie ihr Zweck koinon symferon d. h. das ist, was jeweils als gemeinsamer Vorteil oder als Allgemeinwohl angesehen wird ${ }^{11}$. Das führt uns zu dem Schluss, dass in beiden Fällen, in der Politik und in der Historia animalium, der Ausdruck zôon politikon auf das Wohl der Gemeinschaft oder der Gemeinde bezogen, also nicht biologisch, sondern politisch verstanden wird.

Wäre das grundlegende Charakteristikum der Gemeinde nichts anderes als ein gemeinsames Leben in der Gemeinschaft, dann wären auch andere Lebewesen zôon politikon. Aber die Gemeinde ist, über eine bloß natürliche Gemeinschaft hinaus, eine Gemeinschaft von zusammen lebenden Lebewesen, die alle anderen Gemeinschaften in sich vereinigt (pasa periechûsa tas allas) ${ }^{12}$. Dass die Gemeinde alle anderen Gemeinschaften aufnimmt oder in sich vereinigt bedeutet, dass sie sich von ihnen wesentlich unterscheidet. Dieser Unterschied besteht darin, dass die Gemeinde unter allen Gemeinschaften am meisten (malista) nach dem gemeinsamen Wohl strebt ${ }^{13}$. In ähnlicher Weise kann man auch vom Menschen sagen, dass er im Unterschied zu den anderen Lebewesen mallon, d.h. mehr oder in höherem Maß politikon zôon ist als andere Lebewesen, da er den logos, Sprache besitzt. Und gerade der logos ist es, der dem Menschen die Möglichkeit gibt, ein gutes Leben zu führen. Den anderen Lebewesen ist dies nicht möglich, da sich ihr Leben nur auf der Ebene der Selbsterhaltung und des Überlebens abspielt. Darin beruht der grundlegende Unterschied zwischen ihnen und dem Menschen.

Das zweite grundlegende Charakteristikum des Menschen, dass er logon echei sei, begründet einen wesentlichen Unterschied zwischen dem

Aristoteles, Pol. III 1279a 34.

Aristoteles, Pol. I 1252a 5-6.

In der Politeia heißt es wörtlich, dass die Gemeinde „am meisten und zu den bedeutendsten von allen Gütern" strebt (malista de kai tû kyriôtatû pantôn) (Aristoteles, Pol. I 1252a 4-5).

П Н Г Н / F O N S I (2016), 145-159 
Menschen und den Herdentieren, die ähnlich wie er fähig sind, in der Gemeinschaft zu leben. Im Unterschied zu den Tieren, die nur Geräusche als Ausdruck des Schmerzes oder der Freude von sich geben können, ist es dem Menschen möglich, vermittels des Logos oder der Sprache zu diskutieren und $\mathrm{zu}$ erläutern, was gut und böse, gerecht und ungerecht, schön oder hässlich ist. Dieses Vermögen (dynamis), das die übrigen Lebewesen nicht besitzen, begabt den Menschen mit einem Sinn für das Gute und Böse, Gerechte und Ungerechte.

Aristoteleś Charakterisierung des Menschen als politisches Wesen umfasst zwei wichtige Momente: Einerseits ist der Mensch durch seine Natürlichkeit dazu bestimmt, in der Gemeinschaft $\mathrm{zu}$ leben, und andererseits geht es ihm um eine bestimmte Qualität des Gemeinschaftslebens. Die Gemeinschaft ist zwar eine Gewähr für das Überleben oder den Lebenserhalt, sie ist aber für den Menschen auch die Grundlage für sein Streben nach einem guten Leben. Dass der Mensch dazu getrieben ist (hormê besitzt), in einer solchen Gemeinschaft (polis) $\mathrm{zu}$ leben, bedeutet jedoch noch keineswegs, dass er wirklich darin leben wird. Er könnte durchaus auch völlig isoliert von den Anderen, oder auf dem Land leben, wenn die Gemeinschaft noch nicht die Stufe der polis erreicht hat. ${ }^{14}$ Insofern hat Aristoteles mit dem Begriff physei politikon zôon jene grundlegende menschliche Fähigkeit (dynamis) im Sinn, die uns, die wir nicht naturgemäß zu Bewohnern der polis werden, unter dem Gesichtspunkt des Vermögens betrachtet, uns dazu zu machen. Dieser Befund trifft auch für seine Behauptung zu, dass der Mensch zôon logon echon ist, d.h. dass ihm die Sprache unveräußerlich zugehört. Und auch das bedeutet keinesfalls, dass der Mensch sofort und auf natürliche Weise befähigt ist zu sprechen, sondern dass er ein Vermögen verkörpert, das sich entwickeln muss und durch Erziehung und Selbstbildung aktualisiert

Dasselbe gilt auch im Fall des Wohls und der Gerechtigkeit. Der Mensch besitzt nämlich die Fähigkeit (dynamis), besser gesagt er stellt die Fähigkeit dar, gut und gerecht zu sein, was nicht notwendigerweise bedeutet, dass er immer gerecht handelt oder gerecht ist. 
werden kann. In beiden Fällen handelt es sich um dynamis, also um eine Möglichkeit, durch die wir bestehen, die wir aber keineswegs besitzen.

Wir können nun zusammenfassend konstatieren, dass die Gemeinde auf der Grundlage der Natürlichkeit des Menschen besteht, dass aber ihre Gestalt und ihre Verfassung (politeia) das Ergebnis einer zwischenmenschlichen Übereinkunft ist. Es handelt sich hierbei um zwei unterschiedliche, aber eng miteinander verknüpfte Momente. Das erste betrifft die Natürlichkeit (auf natürliche Weise werden wir vom Leben in Gemeinschaft angezogen) und das zweite berührt die menschlichen Fähigkeiten oder die Kunst (durch die Kunst entfalten wir die menschliche Natürlichkeit). Dieses zweite Moment, das die menschliche Kunstfertigkeit ( technê) betrifft, ist „,künstlich“ und weist in gewisser Hinsicht gemeinsame Charakterzüge mit dem Gemeinschaftsvertrag auf, von dem es sich jedoch durch seine Beziehung zur Natur unterscheidet.

In der Antike sind es die Sophisten, die als erste über den Ursprung und die Entstehung der Gesellschaft debattieren und dabei von einer Antinomie zwischen physis und nomos ausgehen. Als eine der Möglichkeiten dieser Entstehung betrachten auch sie den „Gesellschaftsvertrag“. Auf diese Bedeutung der Sophisten als der antiken Vorläufer der Theorie des Gesellschaftsvertrages hat Charles Kahn in seiner bekannten Studie The Origins of Social Contract Theory aus dem Jahr 1981 aufmerksam gemacht. ${ }^{15}$ Für uns folgt daraus, dass uns das erneute Interesse am Gesellschaftsvertrag, das seit der Veröffentlichung von Rawls Theorie der Gerechtigkeit (1972) zu beobachten ist, nicht nur zu einer Rückkehr zu Denkern wie Hobbes, Locke oder Rousseau führen sollte, sondern bis zu den sophistischen Denkern und von hier aus weiter zu Demokrit, Epikur und Lukrez. Die Konzeptionen des 17.-18. Jahrhunderts und die des 5. -4. Jahrhunderts vor Christus haben vor allem die Voraussetzung der menschlichen Autonomie, sowie die Vorstellung eines ursprünglichen

${ }^{15}$ C. H. KAHN, The Origins of Social Contract Theory, in: G. B. Kerferd (ed.), The Sophists and their Legacy, Wiesbaden 1981, S. 92-108. 
Naturzustandes gemeinsam, der noch keine politisch organisierte Gemeinschaft darstellt. ${ }^{16}$ Individuen sind demnach im natürlichen Zustand Unsicherheit und Bedrohungen ausgesetzt, gegen die sie sich durch die Konstitution von Recht und Gesetz oder durch den Abschluss des Gesellschaftsvertrages, d.h. durch ein Leben in Gemeinschaft zur Wehr setzen.

Aus dem 5.-4. Jahrhundert sind einige Textfragmente überliefert, in denen die Autoren die Rechtskonstitution, die Annahme von Gesetzen und auch die Gesellschaftsverträge beschreiben ${ }^{17}$. Eines der ersten Fragmente, das den natürlichen Zustand und die darauf folgende Rechtskonstitution behandelt und gewöhnlich den Titel Sisyphos trägt ${ }^{18}$, wurde früher Kritias, heute Euripides zugeschrieben ${ }^{19}$. Darin entledigt sich der Mensch der Fesseln der Natur (physis) durch die Erfindung des Gesetzes (nomos) und des Rechts (dikê). Einen ähnlichen Gedanken finden wir in einem bei Iamblichos überlieferten, anonymen Text, der wohl aus dem Jahr 430 v. Chr. stammt ${ }^{20}$. Der Naturzustand wird darin als Zustand beschrieben, in

${ }^{16}$ Im Naturzustand gibt es weder Recht, noch Gerechtigkeit, oder Werte. Von ihnen können wir erst mit der Entstehung der Gesellschaft sprechen, wenn die Menschen zusammenarbeiten, Verhaltensrichtlinien formulieren und bedingt Werte definieren.

17 Siehe z. B. den Vertrag als Gesetzesgrundlage bei Antiphon ( $D K$ B 44a). Das Wort Vertrag (synthêkê) erscheint aber zum ersten Mal in Platons Dialog Kriton (52c), wo das Gesetz Sokrates auffordert, sich dazu zu äußern, ob er es vernichten möchte, wenn er vor Vereinbarungen und Verträgen flüchtet, die es mit ihm abgeschlossen hat. In diesem Dialog taucht auch der Ausdruck homologia oder homologein auf, der Vertrag oder Vereinbarung bedeutet. Das Vorkommen des Infinitivs synthesthai ist z. B. in Platons Politeia belegt (359a 1-b 3).

18 DK 88 B 25. Das Fragment ist Bestandteil des Satyrspiels Sisyphos und wird von der Hauptfigur Sisyphos vorgetragen, daher trägt dieser Teil den angeführten Titel.

19 A. DiHLE hat das Fragment als erster Euripides zugeschrieben (Das Satyrspiel ,Sisyphos; «Hermes»105 [1977], S. 28-42). Zur Geschichte der Autorenschaft des Fragments vgl. C. H. KaHn, The Origins of Social Contract Theory, S. 97.

20 DK 98 (89 A 1-7). Der Text hat wurde in Iamblichoś Werk Protreptikos überliefert (Kap. 20). 
dem die Menschen nicht in der Lage sind, eigenständig zu leben. Die Notwendigkeit (anagkê) führt sie also zu einem gemeinschaftlichen Leben, das sie mit Hilfe des Gesetzes (nomos) und des Rechts (to dikaion) gestalten (DK 89 A 6,1).

Nicht nur die sophistischen Texte, sondern auch die Texte Demokrits und Epikurs $^{21}$ verbindet der Gedanke der menschlichen Unselbstständigkeit, die verschiedene Formen hat, die von der Schwäche und Unfähigkeit des Menschen sich im Naturzustand vor Raubtieren oder vor anderen Menschen zu schützen, bis zur fehlenden Eigenständigkeit bei der Nahrungsbeschaffung reichen. Sie wird durch das Austreten aus dem ursprünglichen Naturzustand und die Gründung einer Gemeinschaft überwunden. In den meisten Fragmenten begründet der Mensch eine Gemeinschaft durch Vertragsabschluss und die Annahme von Gesetzen ${ }^{22}$.

Denselben Gedanken finden wir auch beim führenden Vertreter der Theorie des Gesellschaftsvertrages im 17. Jahrhundert, bei Thomas Hobbes. Während seines französischen „Exils“ lernt er durch die Vermittlung Gassendis den Atomismus Epikurs und auch seinen Gedanken des Gesellschaftsvertrages kennen. Durch diesen hellenistischen Denker inspiriert, entwickelt er die Konzeption des Staates als Kunstprodukt. Der Staat ist also für Hobbes - im Unterschied von Aristoteleś, in der menschlichen Natürlichkeit (physis) begründeten Auffassung und ähnlich wie bei den Sophisten - durch eine Vereinbarung oder einen Vertrag verfasst (nomos). David Keyt nimmt in diesem Zusammenhang an, dass Aristoteles Hobbes darin zustimmen könne, dass die politische Gemeinschaft ein Kunstprodukt sei, da dies sich auch aus den Prinzipien des Aristoteles ergebe. Er stützt sich mit dieser Auffassung

Vgl. A. HavlíčEK, Péče o duši u Démokrita, «Reflexe» 23 (2002), S. 5-22.

In diesen verschiedenartigen Texten finden wir auch viele gemeinsame Ausdrücke oder bildliche Äußerungen, wie zum Beispiel den Ausdruck sporadên, also dass die Menschen verstreut lebten, oder das Bildnis des Naturzustandes, als die Menschen den Tieren ähnlich waren und zwischen ihnen Kampf herrschte, usw. 
auf Passagen in der Politik, wo der Gesetzgeber als Handwerker der Gesetze und Einrichtungen, oder der Verfassungen ausgelegt wird ${ }^{23}$. Durch seine politische Kunst verleiht er der Gemeinde die Form, d. h. die Einrichtung oder Verfassung ${ }^{24}$, und daher ist die Gemeinde hinsichtlich ihrer Form ein künstliches Werk. Aristoteles behauptet aber zugleich auch, dass die Gemeinde physei existiert, d.h. auf einer natürlichen Grundlage. Besteht hier ein Widerspruch, wie Keyt behauptet, oder unterliegt er selbst einem Missverständnis über die Auffassung des Aristoteles? Und kann man die Staatskonzeption von Aristoteles und Hobbes als gegensätzlich erachten?

Beginnen wir zunächst mit der bekannten Äußerung von Hobbes in der Einleitung zum Leviathan:

Denn durch Kunst wird jener grosse Leviathan geschaffen, genannt Gemeinwesen (commonwealth) oder Staat ( state), auf lateinisch civitas, der nichts anderes ist als ein künstlicher Mensch, wenn auch von größerer Gestalt und Stärke als der natürliche, zu dessen Schutz und Verteidigung er ersonnen wurde. For by Art is created that great LEVIATHAN called a COMMON-WEALTH, or STATE (in latine CIVITAS) which is but an Artificiall Man ; though of greater stature and strength than the Naturall, for whose protection and defence it was intended...). ${ }^{25}$

In Hobbes' Schrift De cive (über den Bürger), die in Paris im Jahre 1642 (die englische Version stammt aus dem J. 1651), also noch vor dem Leviathan (engl. 1651) verfasst wurde, kann man im ersten, „Freiheit“ genannten Teil lesen, dass die Mehrzahl Jener, die über die Gesellschaft oder den Staat (commonwealth) geschrieben haben, voraussetzten, dass der Mensch von Geburt an ein zum Leben in der Gemeinschaft geeignetes

Aristoteles, Pol. VII 1325b 40-1326a 5; II 1273b 32-1274b 18.

Aristoteles, Pol. III 1276b 1-11.

T. Hobbes, Leviathan (Lev.), Einführung (Übersetzung I. FeTSCHER, in: T. HOBBES, Leviathan, Frankfurt a. M. 1996); vgl. auch Lev. XVII, 13. 
Lebewesen ist (That Man is a Creature born fit for Society) ${ }^{26}$. In der Anmerkung zum Wort „geeignet“ ( fit) gesteht Hobbes ein, dass die Menschen unter dem Einfluss der Natürlichkeit den Kontakt untereinander suchen oder zusammenkommen (als Beispiel führt er Kinder und Nichtwissende an, die Hilfe von Anderen benötigen). Er ist jedoch davon überzeugt, dass die bürgerliche Gesellschaft (civill society) nicht bloß im Kontakt (meeting) zwischen den Menschen besteht, sondern dass sie auch einen Bund (bond) darstellt, zu dessen Einrichtung Bürgschaften und Verträge notwendig sind ${ }^{27}$.

Hobbes unterscheidet also strikt zwischen einem vorvertraglichen und dem auf einem Vertrag begründeten Zustand. Bloß ein abgeschlossener Vertrag macht aus den Menschen politische Wesen:

Ist dies geschehen, so nennt man diese zu einer Person vereinte Menge Staat, auf lateinisch civitas. (Right to him, and Authorise all his Actions in like manner. This done, the Multitude so united in one Person, is called a COMMONWEALTH, in latine CIVITAS ${ }^{28}$ ( $\mathrm{Lev}$. XVII 13)

\section{Ähnlich heißt es in De cive:}

Der Mensch wird nicht von Natur, sondern durch Zucht zur Gesellschaft geeignet. Ja selbst wenn der Mensch von Natur nach der Gesellschaft verlangte, so folgte doch nicht, daß er von Natur zur Eingehung der Gesellschaft auch geeignet sei; denn das Verlangen und die Fähigkeit sind verschiedene Dinge. (De cive, I 2, Anm. 1)

Man is made fit for Society not by Nature, but by Education: furthermore, although Man were born in such a condition as to desire it, it followes not, that he 
therefore were Born fit to enter into it; for it is one thing to desire, another to be in capacity fit for what we desire. (Kursiv. A. H.) (De cive, I 2, pozn. $)^{29}$

Durch Vertragsabschluss eines Jeden mit Jedem und durch die Übergabe von Vollmachten an eine einzige Person (Souverän), die Jeden zum Autor seiner Taten erklärt, entsteht ein politischer Staat (political commonwealth) ${ }^{30}$ und wird der Mensch $\mathrm{zu}$ einem politischen Lebewesen $^{31}$. Den Grund dafür, dass wir diesen Vertrag abschließen, sieht er in der Erhaltung des Lebens und der Sicherung eines zufriedeneren Lebens ${ }^{32}$.

Darin besteht für Hobbes das hauptsächliche Lebensziel des Menschen, das aus seiner Natürlichkeit, d.h. dem Streben des Individuums nach Erfolg und in der Konsequenz nach Erhaltung des eigenen Lebens resultiert. Dass Hobbes dabei auch die Beziehungen zu den anderen Menschen erwähnt, wie zum Beispiel die Hilfe für Kinder und Blinde, hängt entschieden nicht mit den altruistischen Tendenzen in seiner Konzeption zusammen, sondern im Gegenteil mit dem Egoismus des Menschen. Denn nur zur Erreichung des eigenen Ziels, d.h. zum Zweck der Selbsterhaltung und eines zufriedeneren Lebens ist es für ihn notwendig, den Kontakt zu den übrigen Menschen aufrecht zu halten. Im Kapitel XIII. des Leviathan über die menschliche Natürlichkeit lesen wir von dieser Beziehung zu den anderen Menschen:

Und wenn daher zwei Menschen nach demselben Gegenstand streben, den sie jedoch nicht zusammen geniessen können, so werden sie Feinde und sind in Verfolgung ihrer Absicht, die grundsätzlich Selbsterhaltung und bisweilen nur Genuss ist, bestrebt, sich gegenseitig zu vernichten oder $\mathrm{zu}$ unterwerfen. (Lev. XIII, 3)

T. Hobbes, De cive, I 2, pozn. 1 .

T. Hobbes, Lev. XVII 13-15.

T. Hobbes, Lev. XVII 13.

T. Hobbes, Lev. XVII 1. 
Hobbes interessiert sich also im Grunde nicht für die Qualität der entstandenen Gesellschaftsordnung, denn seine Hauptforderung ist die Erhaltung des Lebens, die durch den Vertragsabschluss und die Regierung des Souveräns erreicht werden soll. Dieser hält die Macht zum Schutz der Menschenleben ganz in seiner Hand ${ }^{33}$. Für den Fall, dass es zu keinem Vertragsabschluss kommen sollte, wäre ein Krieg Aller gegen Alle unvermeidlich. Der grundlegende Schritt, der den Dauerzustand dieses Krieges beendet ${ }^{34}$, ist die freiwillige Unterwerfung der Menschen unter einen von ihnen (oder unter die Volksversammlung), der die Aufgabe hat, sie vor den anderen Menschen zu beschützen ${ }^{35}$.

\section{Zusammenfassung}

Die Staatskonzeption beider Autoren beeinflusst in wesentlicher Weise ihre Anthropologie und konkret ihre Antwort auf die Frage nach dem Ziel und dem Sinn des menschlichen Lebens. Wie wir schon früher angeführt hatten, zählt nach Aristoteles zum Wesen des Menschen nicht nur, dass er physei zôon politikon ist, sondern auch, dass er logos, Sprache besitzt. Ohne die Welt der Sprache könnte der Mensch nicht gut oder schlecht, gerecht oder ungerecht handeln. Die Sprache ist eine notwendige Bedingung dafür, dass der Mensch zu einem politischen Wesen wird ${ }^{36}$. Nur in der Welt der Sprache können wir ein gutes oder ein schlechtes Leben führen. Nach Höffe sollten wir jedoch bei der Auslegung von Aristoteles nicht nur eine der Standardformeln durchsetzen, denen zufolge der Mensch ein vernünftiges oder ein politisches Wesen ist, sondern zwischen ihnen eine bestimmte Art einer dialektischen Beziehung

T. Hobbes, Lev. XVIII 13.

T. Hobbes, Lev. XIII 8.

T. Hobbes, Lev. XVII 13.

Vgl. B. YACK, Problems of a Political Animal, Berkeley-Los Angeles-London 1993, S. $70 \mathrm{nn}$. 
$\operatorname{sehen}^{37}$. Anders wäre dem, wenn der Mensch Gott wäre, denn dann würde seine höchste Glückseligkeit in einer kontemplativen Tätigkeit bestehen. Da aber der Mensch durch sein Wesen dazu bestimmt ist, mit den Anderen zusammen zu leben, ist seine Glückseligkeit als Mensch an die Gemeinde geknüpft und beruht insofern auf moralischen Tugenden ${ }^{38}$. Da aber das bloße Wissen um die Tugenden zur Glückseligkeit nicht ausreicht, müssen wir gut handeln, Gutes tun und gut werden ${ }^{39}$. In diesem Sinn gibt Aristoteles weder in seiner Nikomachischen Ethik (X. Buch), noch in der Politik ${ }^{40}$ einseitig nur dem theoretischen oder dem praktischen Leben den Vorrang, sondern er hält beide in einer dialektischen Spannung.

Bei Hobbes ist - wie wir gezeigt haben - die Situation ganz anders, da für ihn das Wesen und Ziel des Menschenlebens darin besteht, sich „der Gefahr der Zerstörung zu widersetzen “41 und d.h., das eigene Leben zu erhalten. Das ist zwar auch der grundlegende und charakteristische Wesenszug, der für Aristoteles die Gemeinde entstehen lässt, aber ihr Fortdauern beruht bei ihm auf ganz anderen Gründen als für Hobbes, da sie aus seiner Sicht wegen des guten Lebens überdauert. In der Darlegung von Hobbes muss dem gegenüber der Staat den Frieden sicherstellen, einheitlich bleiben und alle Bürger sind der Meinung des Souveräns verpflichtet ${ }^{42}$.

Es ist nötig, den Unterschied zwischen Aristoteles und Hobbes darin zu suchen, dass beide Denker eine andere Ansicht der Grundlage hatten,

Buch V., wo die beste Gemeinde einem Menschen zugeordnet wird, über den Alle einstimmig sagen: „das ist meines, das ist nicht meines“ (462c).

П Н Г Н / F O N S I (2016), 145-159 
aus dem die Gemeinschaft entspringt und sich bildet. Für Aristoteles ist ähnlich wie für Platon die Grundlage der Mensch, der durch seine Natürlichkeit nach dem eigenen Lebenserhalt strebt. Aristoteles und Hobbes, setzen in gewissem Sinn einen Naturzustand voraus, der für den Einen der Zustand des Vermögens (dynameis), für den Anderen der Kriegszustand ist, aus dem er durch Vertragsabschluss entkommt. Durch Aristoteles wird die Natürlichkeit wie auch das Vermögen durch Kunst (technê) erfüllt und verwirklicht, wenn jedes Individuum je nach seiner Geschicklichkeit und Fähigkeit unterschiedliche Stufen der Erfüllung erreicht. Man kann insofern sagen, dass erst die Kunst der Natürlichkeit Gestalt verleiht. Im Gegensatz dazu bewertet Hobbes den Naturzustand des dauerhaften Krieges Aller gegen Alle negativ und ersetzt ihn daher durch einen neuen, und zwar friedlichen oder vertraglichen Zustand. In diesem Fall handelt es sich nicht darum, die Natürlichkeit zu vollenden oder umzugestalten, sondern den natürlichen Zustand durch einen künstlichen $\mathrm{zu}$ ersetzen. Ein weiterer Unterschied zwischen beiden Denkern besteht darin, dass es Hobbes um die Entstehung des Staates und folglich um seine Einheit geht, die durch die Zustimmung Aller erreicht wird, wobei die Meinung eines Jeden mit der Meinung des Souveräns übereinstimmen muss. Demgegenüber interessiert Aristoteles der Sinn oder das Ziel der Gemeinde, das ihr nicht vorgegeben ist, sondern Gegenstand ihrer internen Diskussion ist. Steht schließlich bei beiden Denkern die Entstehung der Gemeinde im Dienst des Lebens, so ist der Zweck ihres Bestehens für Hobbes dessen Erhaltung, für Aristoteles hingegen ein gutes Leben. 